\title{
Detection of complex formation and determination of intermolecular geometry through electrical anharmonic coupling of molecular vibrations using electron-vibration-vibration two-dimensional infrared spectroscopy
}

\author{
Rui Guo ${ }^{a}$, Frederic Fournier ${ }^{a, b}$, Paul M. Donaldson ${ }^{a, b} \dagger$, Elizabeth M. Gardner $^{a}$, Ian R. Gould ${ }^{a}$, \\ and David R. Klug ${ }^{a, b}$ \\ ${ }^{a}$ Department of Chemistry, Chemical Biology Centre, ${ }^{b}$ Single Cell Proteomics Project, Imperial \\ College London, London SW7 2AZ, United Kingdom
}

†Present address: Physikalisch-Chemisches Institut, Universität Zürich, Winterthurerstrasse 190, 8057 Zürich, Switzerland

Electrical interactions between molecular vibrations can be nonlinear and thereby produce intermolecular coupling even in the absence of a chemical bond. We use this fact to detect the formation of an intermolecular complex using electron-vibration-vibration two-dimensional infrared spectroscopy (EVV 2DIR) and also to determine the distance and angle between the two molecular species.

One of the great strengths of NMR has been its ability to measure the coupling strength between nuclear spins and from this to deduce the distance between them. This capability stems from the relatively simple physics that determines the spin-spin coupling, which allows distances to be inferred ${ }^{1}$. In essence the coupling follows a 
dipolar distance dependence. The apparent similarities between 2D NMR methods and its optical analogues such as 2DIR have fueled the hope that geometric information might also be obtained from these techniques. It is certainly true that structural analysis, rather than structural determination, can be greatly assisted by 2DIR methods, as the signals, in the form of cross peaks, can be very sensitive to molecular structure and vibrational cross peaks can be accurately calculated from known structures ${ }^{2-5}$. When it comes to structural determination however, despite some progress over recent years ${ }^{5,6}$, this hope has not yet been fulfilled. This is partly due to the more complex coupling physics that connects molecular vibrations with each other when compared with the Hamiltonians that are responsible for nuclear spin coupling. In essence there are two types of anharmonicities that result from vibration-vibration coupling: mechanical and electrical. When chemical bonds are present it is usual that mechanical anharmonicity dominates. There is currently no known way to map mechanical anharmonicity onto molecular geometry and therefore no known way to use 2DIR methods to determine molecular structures. We show here however there is an important class of molecular systems for which structural analysis is possible, namely those systems in which electrical anharmonicity dominates. This class of problems include those of intermolecular interactions and molecular complexation, prevalent in supramolecular self-assemblies and many biological systems ${ }^{7}$.

EVV 2DIR spectroscopy is a coherent multidimensional spectroscopy first developed by Wright $^{8}$, suggested by $\mathrm{Cho}^{4}$, and further developed in our group ${ }^{2,3,9,10}$. Unlike other 
forms of 2DIR spectroscopy ${ }^{11}$, it has the unique feature of being inherently sensitive to electrical anharmonicity. We show here that for systems in which electrical anharmonicity dominates, the coupling can be well described by the physics of multipole coupling which, for the example given in this paper, is approximated by dipole-dipole coupling. We also show that for the case of a complex-forming molecular system, phenylacetylene (PA) and benzonitrile $(\mathrm{BN})$, the cross peaks formed by dipolar couplings appear with the predicted strength and at the predicted locations in an EVV 2DIR spectrum. Finally we show how the polarization dependence of the cross peaks can be used to deduce the angle between the molecules in question and thereby estimate the distances between the two interacting modes via the intensities of the intermolecular cross-peaks.

There are two types of anharmonicities intrinsic to a molecular system. The first of these comprises the lowest-order anharmonic terms in molecular potential energy surface, known as the mechanical anharmonicity, while the second is the electrical anharmonicity, defined as the nonlinearity $\left(\frac{\partial^{2} \boldsymbol{\mu}}{\partial Q_{i} \partial Q_{j}}\right)$ in molecular electrical dipole moment ${ }^{12}$. Every implementation of 2DIR spectroscopy depends in one way or the other on these two anharmonicities for its signals, but a unique feature of EVV 2DIR spectroscopy is its sensitivity to electrical anharmonicity even in the absence of mechanical anharmonicity. This makes it possible to use EVV 2DIR spectra for the determination of relative geometries of interacting chemical groups. Consider a molecular system composed of two or more chemical groups, which, though not directly bonded to each other, lie close enough to interact. The vibrations of each 
chemical group are usually localized on the group, and the coupling between two such vibrations can only take place via through-space electrostatic interactions. In principle this coupling can be anharmonic, thereby leading to EVV 2DIR cross peaks. Numerous candidates for this kind of systems can be found in published X-ray crystal structures of proteins in the form of interacting side chains, such as the F19-Y63 pair in $\mathrm{p} 53-\mathrm{mdm} 2$ complex $^{13}$. Through-space electrostatic interactions between two chemical groups can also lead to mechanical anharmonicities, however this effect is known to be much weaker. As shown by $\mathrm{Cho}^{14}$, mechanical anharmonicity induced by two interacting dipoles involves, as a prerequisite, second-order dipole moment derivatives which are usually small in magnitude. On the other hand, electrical anharmonicity from this same dipole-dipole interaction only involves first-order derivatives of dipole moments and polarizabilities (vide infra). Thus it is reasonable to assume that through-space-induced mechanical anharmonicity is negligible compared with through-space-induced electrical anharmonicity from which EVV 2DIR signals arise entirely. Later in this paper we also provide experimental verification of this theoretical assumption.

For two uncharged chemical groups weakly interacting with each other, each carrying one vibrational mode (labeled as 1 and 2), the total Hamiltonian of the pair can be written as a sum of the non-interacting Hamiltonians of the monomers and an interaction potential $H^{\prime}$. If we take an approximation that the interaction between the pair is mostly dipolar, then $H^{\prime}$ can be written as $H^{\prime}=-\boldsymbol{\mu}_{1} \mathbf{T}_{12}^{(2)} \boldsymbol{\mu}_{2}$ following Buckingham $^{15} . \boldsymbol{\mu}_{i}$ is the dipole moment of group i. $\mathbf{T}_{12}^{(2)}=\left(3 \mathbf{R} \mathbf{R}-\mathbf{I} R^{2}\right) / R^{5}$ is the 
interaction tensor, with $\mathbf{R}$ being the distance vector pointing from group 1 to 2 and $R=|\mathbf{R}|$. After taking derivatives of the total energy of the system with respect to external electric field and normal mode coordinates $Q_{1}$ and $Q_{2}$, an expression for the electrical anharmonicity induced by dipole-dipole interaction between the two vibrations can be written as

$$
\left(\frac{\partial^{2} \boldsymbol{\mu}}{\partial Q_{1} \partial Q_{2}}\right)_{\text {ind }}=\frac{\partial \boldsymbol{\alpha}_{1}}{\partial Q_{1}} \cdot \mathbf{T}_{12}^{(2)} \cdot \frac{\partial \boldsymbol{\mu}_{2}}{\partial Q_{2}}+\frac{\partial \boldsymbol{\mu}_{1}}{\partial Q_{1}} \cdot \mathbf{T}_{12}^{(2)} \cdot \frac{\partial \boldsymbol{\alpha}_{2}}{\partial Q_{2}}
$$

$\boldsymbol{\alpha}_{i}$ is the polarizability tensor of group $i$. Information about the interaction geometry of the two chemical groups is incorporated in the induced electrical anharmonicity by the interaction tensor $\mathbf{T}_{12}^{(2)}$. As shown by Okumura et al. ${ }^{16}$, Cho ${ }^{14}$ and Hahn et al. ${ }^{17}$, electrical anharmonicity should be inversely proportional to $R^{3}$, the same conclusion can be reached here.

The complete procedure of calculating the third-order nonlinear susceptibility $\chi^{(3)}$ of EVV 2DIR signals in the presence of mechanical and electrical anharmonicities has been given by Kwak et $\mathrm{al}^{4}$. However, when there is only electrical anharmonicity involved, $\chi^{(3)}$ can be written in a much simpler form of vector/tensor products as

$$
\chi_{\mathrm{PPP}}^{(3)} \propto \operatorname{Tr}\left(\frac{\partial \boldsymbol{\alpha}}{\partial Q_{2}}\right)\left(\frac{\partial^{2} \boldsymbol{\mu}}{\partial Q_{1} \partial Q_{2}} \cdot \frac{\partial \boldsymbol{\mu}}{\partial Q_{1}}\right)+2\left(\frac{\partial \boldsymbol{\alpha}}{\partial Q_{2}} \cdot \frac{\partial^{2} \boldsymbol{\mu}}{\partial Q_{1} \partial Q_{2}}\right) \cdot \frac{\partial \boldsymbol{\mu}}{\partial Q_{1}}
$$

with mode 1 as the $\omega_{\alpha}$ mode. Tr indicates the trace of the tensor. Eq. (2) is for a polarization configuration with all the electric fields of the three laser pulses parallel to each other and in the plane of propagation ${ }^{2}$ (denoted as PPP) and includes orientation averaging for an isotropic media. Another polarization configuration in EVV 2DIR spectroscopy with the polarization plane of the visible laser pulse 
perpendicular to the other two is denoted as PPS, for which $\chi^{(3)}$ can be expressed as

$$
\chi_{\mathrm{PPS}}^{(3)} \propto 2 \operatorname{Tr}\left(\frac{\partial \boldsymbol{\alpha}}{\partial Q_{2}}\right)\left(\frac{\partial^{2} \boldsymbol{\mu}}{\partial Q_{1} \partial Q_{2}} \cdot \frac{\partial \boldsymbol{\mu}}{\partial Q_{1}}\right)-\left(\frac{\partial \boldsymbol{\alpha}}{\partial Q_{2}} \cdot \frac{\partial^{2} \boldsymbol{\mu}}{\partial Q_{1} \partial Q_{2}}\right) \cdot \frac{\partial \boldsymbol{\mu}}{\partial Q_{1}} .
$$

The expression of $\chi^{(3)}$ in the form of dot-products as in Eqs. (2) and (2') underlines the dependence of signal intensity on the directional relationships between the two interacting chemical groups. Together with Eq. (1), they link EVV 2DIR signal intensities to the interaction geometry between the two chemical groups, embedded in the interaction tensor $\mathbf{T}$ and the dot-products. The observables in these experiments are spectral properties, such as transition dipoles etc. Therefore the "directional relationships" mentioned above are those between transitional properties of the interacting groups, thus to obtain molecular geometries we also need to know the orientation of molecular transition dipoles relative to the molecular axes.

We applied the above model to the case of two linear chemical groups and the coupling between their stretching modes. FIG. 1(a) defines the structural parameters in this case. Apart from the distance $R$, three angles, $\theta_{1}, \theta_{2}$, and $\phi$, are needed to define the spatial relationship between the two groups, with $\phi$ defined as the angle between the two transition dipoles. By choosing an appropriate coordinate system, Eq. (1) can be used to obtain the Cartesian components of the induced electrical anharmonicity. However even for this special case, the resultant expressions are complicated. For simplicity, since the longest axis of a transition polarizability tensor roughly lines up with the molecular axis in a linear system, the smaller components of the tensor are ignored. The induced electrical anharmonicity can then be expressed in a simple form, and the effect of the smaller components can be corrected post hoc as 
will be shown later. With this approximation, the magnitude of induced electrical anharmonicity can be obtained from Eq. (1) as

$$
\left|\frac{\partial^{2} \mu}{\partial Q_{1} \partial Q_{2}}\right|_{\text {ind }}=\frac{\cos \phi+3 \cos \theta_{1} \cos \theta_{2}}{R^{3}}\left(k^{2}+1+2 k \cos \phi\right)^{1 / 2} \frac{\partial \alpha_{1}}{\partial Q_{1}} \frac{\partial \mu_{2}}{\partial Q_{2}} .
$$

$k$ is a dimensionless constant defined as: $k=\left(\frac{\partial \alpha_{2}}{\partial Q_{2}} \frac{\partial \mu_{1}}{\partial Q_{1}}\right) /\left(\frac{\partial \alpha_{1}}{\partial Q_{1}} \frac{\partial \mu_{2}}{\partial Q_{2}}\right)$. Using Eqs. (1), (2) and (2'), one can obtain an expression explicitly connecting $\chi^{(3)}$, and thus cross peak intensity, with the geometrical parameters defined in FIG. 1(a). In particular, the signal ratio of cross peak intensities measured at PPS and PPP configurations is

$$
\left|\chi_{\mathrm{PPS}}^{(3)} / \chi_{\mathrm{PPP}}^{(3)}\right|^{2}=\left|\left(2+k \cos \phi-\cos ^{2} \phi\right) /\left(1+3 k \cos \phi+2 \cos ^{2} \phi\right)\right|^{2} .
$$

This provides us a convenient way to determine $\phi$ from the signal ratio between PPP and PPS polarisation configurations. The distance $R$ between the two groups can then be calculated from the absolute intensity of the signal, which in practice can also be determined by comparison with an intramolecular coupling of known strength.

The dipole-dipole coupling model was used to calculate the EVV 2DIR spectrum for a dimer formed by $\mathrm{BN}$ and $\mathrm{PA}$. The coupled vibrations are the $\mathrm{CC}$ and $\mathrm{CN}$ stretching modes. Ab initio geometry optimization for the dimer at MP2(Full)/6-31+G(d,p) level with BSSE correction ${ }^{18}$ led to a configuration as shown in FIG. 1(b), similar to the displaced parallel configuration of the benzene dimer ${ }^{19}$. The bonding energy was calculated to be about $4.4 \mathrm{kcal} / \mathrm{mol}$, which is comparable to that for $\mathrm{BN}$ and benzene ${ }^{20}$, indicating that $\pi-\pi$ interaction between the two phenyl rings is responsible for the complexation of $\mathrm{BN}$ and PA. The distance between $\mathrm{CC}$ and $\mathrm{CN}$ bond centres is about 
3.68 A. For the two monomers BN and PA, first-order derivatives of dipole moments and polarizability were calculated separately from their optimized geometries and listed in TABLE I, from which $k$ is calculated to be 2.65. Then Eq. (1) was used to calculate the induced electrical anharmonicity between the two modes, also given in TABLE I. Note for this result the full transition polarizability tensors were used, which when compared with another calculation using only the largest tensor components will enable us to correct the effect of the smaller tensor components vide infra. From the resultant electrical anharmonicity, an EVV 2DIR spectrum was calculated and combined with the calculated monomeric 2DIR spectra of BN and PA to obtain the EVV 2DIR spectrum for the BN-PA dimer as shown in FIG.1(c). The cross peak at $2113 / 4226 \mathrm{~cm}^{-1}$ is between CC stretching fundamental and first overtone (CC-CC cross peak), and similarly the one at $2239 / 4478 \mathrm{~cm}^{-1}$ is between $\mathrm{CN}$ stretching fundamental and first overtone ( $\mathrm{CN}-\mathrm{CN}$ cross peak). Most significantly, the calculation predicts two new cross peaks at $2113 / 4352$ and $2239 / 4352 \mathrm{~cm}^{-1}$ (CC-CN and $\mathrm{CN}-\mathrm{CC}$ cross peaks), arising entirely from the electrical anharmonicity induced by through-space interaction between $\mathrm{CC}$ and $\mathrm{CN}$ groups.

To verify our theoretical predictions, EVV 2DIR spectra of pure liquid BN, pure liquid PA and a 50\%-50\% liquid mixture of them were measured at room temperature and shown in FIG. 2(a). A description of our EVV apparatus and other experimental details can be found elsewhere ${ }^{2,3,9,10}$. Compared with the spectra of pure liquids, two new cross peaks were present at $2110 / 4335$ and $2225 / 4335 \mathrm{~cm}^{-1}$ in the spectrum of the BN-PA mixture, as predicted by theory. The fact that the pair of cross peaks do not 
exist in either of the two pure liquids, makes it unambiguously clear that they must come from the interaction between BN and PA. For comparison the FTIR spectrum of the BN-PA mixture as shown in FIG. 2(b), is as expected almost exactly the sum of the two pure liquids and betrays no traces of the intermolecular coupling. It is expected that the intermolecular coupling should be visible by the presence of a weak intermolecular combination band in the near infra-red. Indeed the formation of the EVV 2DIR spectrum requires excitation of this band by one of our IR pulses. In fact this band is both extremely weak, and the spectrum contains sufficient other combination bands and overtones that the intermolecular combination band is not reliably identifiable or assignable from FTIR data.

For the three spectra in FIG. 2(a), the pulse ordering used is that with the $\omega_{\alpha}$ as pulse 1 in the pulse sequence. As a proof of our assumption that mechanical anharmonicity in BN-PA complexation is negligible, the pulse ordering was reversed as discussed in more detail in our previous papers ${ }^{2,3}$. If the anharmonicity is purely electrical, then the observed CC-CN and $\mathrm{CN}-\mathrm{CC}$ cross peaks should disappear upon reversing the pulse ordering. This is confirmed in the spectra of FIG. 2(c). Thus we can conclude with confidence that the intermolecular mechanical anharmonicity is indeed negligible in this system.

Although in a liquid mixture of $\mathrm{BN}$ and PA, multiple interactions between several BN and PA molecules may exist to some extent, the gas-phase dimer as depicted in FIG. 1(b) was used as a starting point following Zheng et $\mathrm{al}^{5}$. We measured the PPS/PPP signal ratios as $0.417 \pm 0.037$ for the $\mathrm{CC}-\mathrm{CN}$ cross peak and $0.368 \pm 0.017$ for the 
$\mathrm{CN}-\mathrm{CC}$ cross peak. To apply Eq. (4) to these ratios requires correction for the effect of the smaller transition polarizability components. This was done by first calculating the deviation of the angle $\phi$ from two theoretical PPS/PPP ratios, one calculated with these smaller components and another without, then correcting the experimental estimation accordingly. The final result is an angle for $\phi$ of $31^{\circ}$ calculated directly from the EVD 2DIR data. In fact, transition dipoles of the $\mathrm{CC}$ and $\mathrm{CN}$ stretching modes of BN-PA dimer can be numerically calculated at B3LYP/6-311++G(d,p) level, and the angle between the two vectors is found to be $17^{\circ}$ thus there is an apparent $14^{\circ}$ error between the calculated and measured molecular geometries. One obvious source of error is the neglected multiple interaction which can only be addressed with a better knowledge of the liquid structure of the system, yet another source of error may come from the thermal distribution of $\phi$ at room temperature, since the relative sliding-rotation between the two aromatic rings in FIG. 1(b) involves only very low potential barriers, hinted by a very-low vibration frequency $\left(\sim 13 \mathrm{~cm}^{-1}\right)$. We note that bonded liquid dimers have previously been studied by other 2DIR methods ${ }^{5}$ and that the approximation of one dominating molecular dimer geometry even in a liquid may not be such a bad approximation.

Determination of distance $R$ between the two interacting groups requires in general the measurement of absolute intensity of cross peaks. However if we use the CN-CN cross peak in the 2DIR spectrum as an internal reference, and the chemical assumption that the interacting BN-PA pair should keep a roughly face-on geometry for efficient $\pi-\pi$ interaction, the absolute intensity of $\mathrm{CN}-\mathrm{CC}$ cross peak can be 
roughly estimated by multiplying the $\mathrm{CN}-\mathrm{CC} / \mathrm{CN}-\mathrm{CN}$ signal ratio with the intensity of the reference peak. With an experimental value of this ratio at about $0.73 \pm 0.05$, calculations gave a distance between the $\mathrm{CC}$ and $\mathrm{CN}$ groups at about $\sim 2.6 \AA$. Although this is shorter than the predicted $a b$ initio distance $(\sim 3.7 \AA)$, considering the crude approximations used throughout this is a surprisingly good result.

As a summary, we have shown the detection of electrically-induced intermolecular cross peaks and subsequent determination of relative geometry of the $\mathrm{CC}$ and $\mathrm{CN}$ vibrations in the BN-PA complex. The acceptable agreement with the ab initio geometry gives us confidence in the dipole-dipole model outlined here and its underlying assumptions. Although we only give explicit expressions connecting EVV 2DIR signal intensity with geometrical parameters for the case of two linear chemical groups, similar expressions for more complicated cases can be readily obtained. Our results have shown that due to its unique sensitivity to electrical anharmonicity, EVV 2DIR spectroscopy can be used as a method for the detection of weakly interacting complexes and also for analysis of their interaction geometry. Though the example in this paper is a simple two-component liquid mixture, there is no reason why the same principle shown here can not be equally applied to more complicated systems. As EVV 2DIR spectroscopy has already been shown to be able to greatly reduce spectral congestion of complicated biological samples ${ }^{3,9,10}$, the ability to detect weak interactions between chemical groups makes it possible in principle to apply this approach to the study of the interaction of amino-acid side-chains. In particular, we foresee a future for this approach both in the detection of intra-protein, protein-protein 
and protein-ligand interactions and in the determination of the geometry of these interactions.

This research was supported by the Engineering and Physical Sciences Research Council (EPSRC) through the Single Cell Proteomics Project (www.singlecellproteomics.ac.uk) and Platform Grant Scheme, the Biotechnology and Biological Science Research Council (BBSRC) through the Chemical Biology Centre Doctoral Training Centre (www.chemicalbiology.ac.uk). 
1. H. Friebolin and J. K. Becconsall, Basic one- and two-dimensional NMR spectroscopy, 4th edn., Wiley-VCH, Weinheim, 2005.

2. P. M. Donaldson, R. Guo, F. Fournier, E. M. Gardner, L. M. C. Barter, C. J. Barnett, I. R. Gould, D. R. Klug, D. J. Palmer and K. R. Willison, J Chem Phys, 2007, 127, 114513-114511

3. P. M. Donaldson, R. Guo, F. Fournier, E. M. Gardner, I. R. Gould and D. R. Klug, Chem Phys, 2008, 350, 201-211.

4. K. Kwak, S. Cha, M. H. Cho and J. C. Wright, J Chem Phys, 2002, 117, 5675-5687.

5. J. R. Zheng, K. Kwak, J. Asbury, X. Chen, I. R. Piletic and M. D. Fayer, Science, 2005, 309, 1338-1343.

6. D. V. Kurochkin, S. R. G. Naraharisetty and I. V. Rubtsov, P Natl Acad Sci USA, 2007, 104, 14209-14214.

7. S. K. Burley and G. A. Petsko, Science, 1985, 229, 23-28.

8. $\quad$ W. Zhao and J. C. Wright, Phys Rev Lett, 1999, 83, 1950-1953.

9. F. Fournier, E. M. Gardner, R. Guo, P. M. Donaldson, L. M. C. Barter, D. J. Palmer, C. J. Barnett, K. R. Willison, I. R. Gould and D. R. Klug, Anal Biochem, 2008, 374, 358-365.

10. F. Fournier, E. M. Gardner, D. A. Kedra, P. M. Donaldson, R. Guo, S. A. Butcher, I. R. Gould, K. R. Willison and D. R. Klug, P Natl Acad Sci USA, 2008, 105, 15352-15357.

11. M. H. Cho, Chem Rev, 2008, 108, 1331-1418.

12. S. Califano, Vibrational states, Wiley, London; New York, 1976.

13. P. H. Kussie, S. Gorina, V. Marechal, B. Elenbaas, J. Moreau, A. J. Levine and N. P. Pavletich, Science, 1996, 274, 948-953.

14. M. Cho, Phys Rev A, 2000, 61, 023406.

15. A. D. Buckingham, Advances in Chemical Physics, 1967, 12, 107.

16. K. Okumura, A. Tokmakoff and Y. Tanimura, J Chem Phys, 1999, 111, 492-503.

17. S. Hahn, K. Kwak and M. Cho, J Chem Phys, 2000, 112, 4553-4556.

18. M. J. Frisch, G. W. Trucks, H. B. Schlegel, G. E. Scuseria, M. A. Robb, J. R. Cheeseman, J. Montgomery, J. A., T. Vreven, K. N. Kudin, J. C. Burant, J. M. Millam, S. S. Iyengar, J. Tomasi, V. Barone, B. Mennucci, M. Cossi, G. Scalmani, N. Rega, G. A. Petersson, H. Nakatsuji, M. Hada, M. Ehara, K. Toyota, R. Fukuda, J. Hasegawa, M. Ishida, T. Nakajima, Y. Honda, O. Kitao, H. Nakai, M. Klene, X. Li, J. E. Knox, H. P. Hratchian, J. B. Cross, V. Bakken, C. Adamo, J. Jaramillo, R. Gomperts, R. E. Stratmann, O. Yazyev, A. J. Austin, R. Cammi, C. Pomelli, J. W. Ochterski, P. Y. Ayala, K. Morokuma, G. A. Voth, P. Salvador, J. J. Dannenberg, V. G. Zakrzewski, S. Dapprich, A. D. Daniels, M. C. Strain, O. Farkas, D. K. Malick, A. D. Rabuck, K. Raghavachari, J. B. Foresman, J. V. Ortiz, Q. Cui, A. G. Baboul, S. Clifford, J. Cioslowski, B. B. Stefanov, G. Liu, A. Liashenko, P. Piskorz, I. Komaromi, R. L. Martin, D. J. Fox, T. Keith, M. A. Al-Laham, C. Y. Peng, A. Nanayakkara, M. Challacombe, P. M. W. Gill, B. Johnson, W. Chen, M. W. Wong, C. Gonzalez and J. A. Pople, Gaussian 03, Revision D.01, Gaussian, Inc., Wallingford CT, 2004.

19. M. O. Sinnokrot and C. D. Sherrill, J Phys Chem A, 2004, 108, 10200-10207.

20. M. O. Sinnokrot and C. D. Sherrill, J Am Chem Soc, 2004, 126, 7690-7697. 


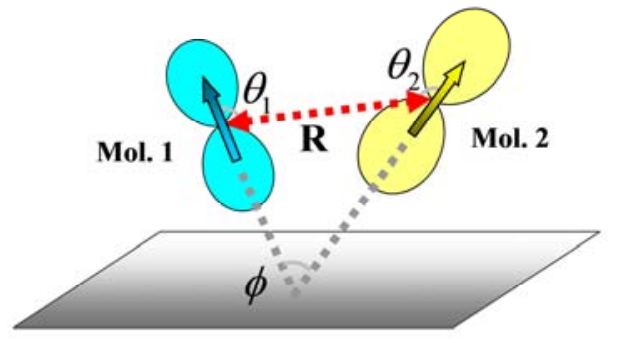

(a)

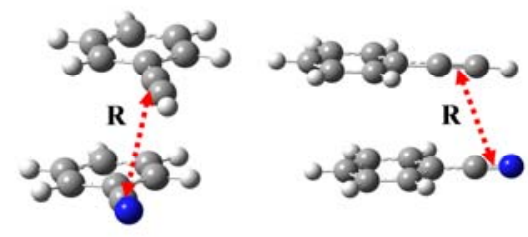

(b)

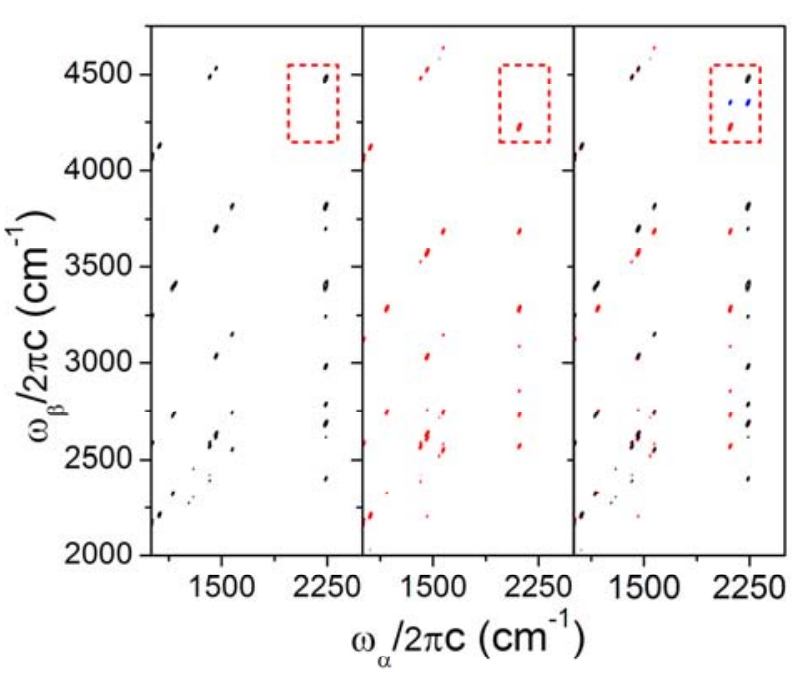

(c)

FIG. 1. (a) Geometrical parameters and molecular properties in the dipole-dipole model used in the text, arrows stand for transition dipoles and lobes transition polarizabilities; (b) BN-PA dimer optimized at MP2/6-31+G(d,p) level with BSSE correction; (c) theoretical EVV 2DIR spectra for BN(left), PA(centre) and BN-PA dimer(right), corresponding experimental spectral regions were encircled by red squares. 

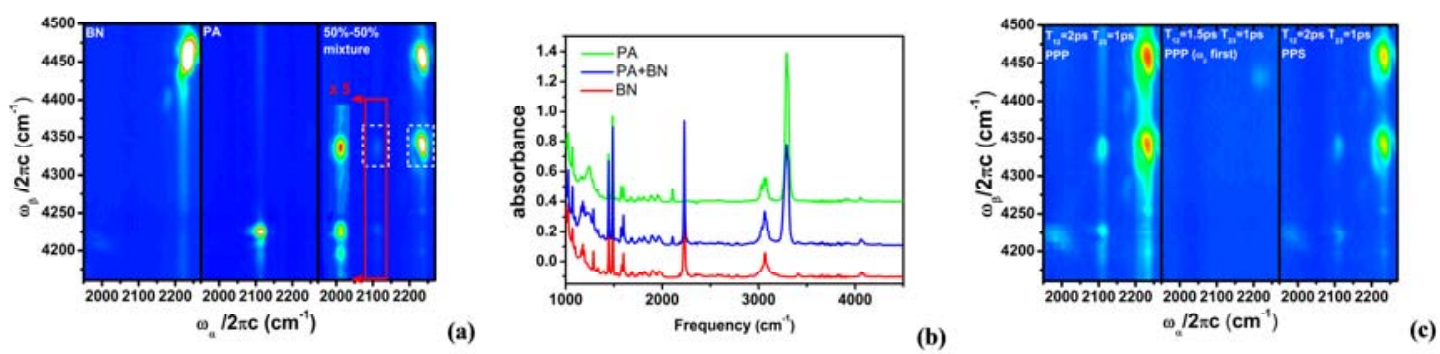

FIG. 2. (a) Experimental EVV 2DIR spectra for pure liquid BN, pure liquid PA, 50\%-50\% liquid mixture of BN and PA; (b) FTIR spectra of the same three samples;

(c) experimental EVV 2DIR spectra for the BN-PA mixture at PPP, PPP with reversed pulse ordering and PPS configurations. 
TABLE I Theoretical parameters used in calculations of the BN-PA system ${ }^{\text {a }}$

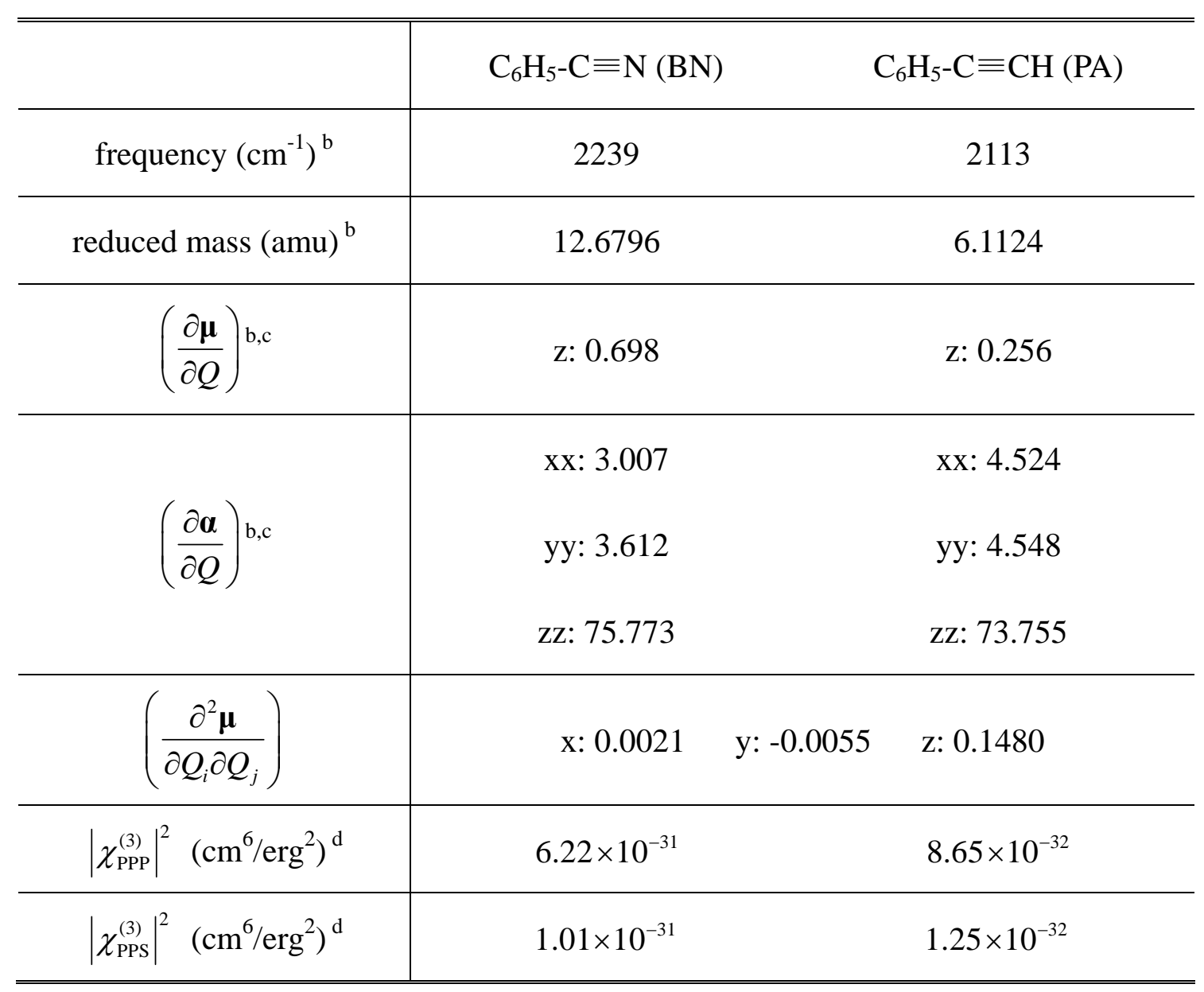

${ }^{\mathrm{a}}$ in a.u. unless stated otherwise.

${ }^{\text {b }}$ calculated at B3LYP/6-311++G(d,p) level.

${ }^{\mathrm{c}}$ in coordinate systems of the monomers, only non-zero Cartesian components were given.

${ }^{\mathrm{d}}$ with CN and CC stretching modes as $\omega_{\alpha}$ respectively. 